\title{
Experimental Investigation of the Effect of Rotor Tip Gaps on 3D Separating Flows inside the Stator of a Highly Loaded Compressor Stage
}

\author{
Baojie Liu ${ }^{1,2,3}$, Guangfeng $\mathrm{An}^{1}$, Xianjun $\mathrm{Yu}^{1,2,3}$, Zhibo Zhang ${ }^{4}$ \\ ${ }^{1}$ School of Energy and Power Engineering, Beihang University, Beijing, China \\ ${ }^{2}$ National Key Laboratory of Science \& Technology on Aero-Engine Aero-Thermodynamics, Beihang \\ University, China \\ ${ }^{3}$ Collaborative Innovation Center of Advanced Aero-Engine, Beihang University, China \\ ${ }^{4}$ AVIC Shenyang Engine Design and Research Institute, Shenyang, China
}

Corresponding author:

Xianjun Yu, Group 404, School of Energy and Power Engineering, Beihang University, Beijing 100191, China

Email: yuxj@buaa.edu.cn

\begin{abstract}
Flow separations in compressor blade passages are common and can cause significant flow blockage and loss production. This paper investigates experimentally the three-dimensional (3D) flow separations in a highly loaded low-speed large-scale compressor facility. Oil flow visualizations, stereoscopic particle image velocimetry (SPIV), and five-hole probe measurements are conducted at certain conditions from the near-chock to near-stall condition along the compressor operating line. The 3D separation and vortex flow structures in the stator at different operating conditions are analyzed. By changing the size of the rotor tip gap, six groups of oil-flow pictures are obtained to study the effect of the rotor gaps on the 3D separating flows in the downstream stator. The variation of the corner separation scale is almost linear with the rotor tip gap size. Along the compressor operating line, four typical 3D flow structures are found inside the stator passage. Between the second typical 3D flow structure and the third typical 3D flow structure, an unstable stage exists on the compressor operating line; during this stage, the hub corner separation becomes an open separation from the closed type. A smaller rotor tip gap corresponds to an earlier unstable stage. Finally, a critical rotor tip blockage state usually existed for the transform of corner separation types at a certain rotor tip gap configuration. This discovery is valuable for the study of multistage compressor matching problems at off-design conditions.
\end{abstract}

Keywords: 3D Separating Flows; Oil flow visualizations; Rotor tip gap; Stereoscopic PIV; Highly loaded compressor 


\title{
Nomenclature
}

\author{
DE compressor design condition \\ NC compressor near-choke condition \\ NS compressor near-stall condition \\ MSPR compressor maximum static pressure rise condition \\ SS blade suction surface \\ PS blade pressure surface \\ $L E \quad$ blade leading edge \\ TE blade trailing edge \\ SL separation line \\ AL attachment line \\ F focus point \\ $S \quad$ saddle point \\ $R e_{\text {chord }} \quad$ Reynolds number based on chord \\ SPIV stereoscopic particle image velocimetry \\ EXP experimental results \\ $P_{\text {in }} \quad$ compressor inlet static pressure $(\mathrm{Pa})$ \\ $P_{\text {out }} \quad$ compressor outlet static pressure $(\mathrm{Pa})$ \\ $V z \quad$ axial flow velocity $(\mathrm{m} / \mathrm{s})$ \\ $U_{\text {mid }} \quad$ rotor speed at the middle span $(\mathrm{m} / \mathrm{s})$ \\ $C p \quad$ static pressure rise coefficient, $\left(P_{\text {out }} P_{\text {in }}\right) /\left(1 / 2 \rho U^{2}{ }_{\text {mid }}\right)$ \\ $C v z \quad$ nondimensional axial velocity, $V z / U m i d$ \\ $C w \quad$ nondimensional velocity component in the streamwise direction \\ $\varphi \quad$ mass flow coefficient, $V z / U_{\text {mid }}$ \\ $\rho \quad$ air density $\left(\mathrm{kg} / \mathrm{m}^{3}\right)$
}




$\begin{array}{ll}\beta & \text { stator inlet flow angle (degree) } \\ B_{m} & \text { blockage coefficient } \\ m_{b} & \text { mass flow rate caused by the blockage } \\ m_{t} & \text { local total mass flow rate } \\ A_{b} & \text { blockage region area }\end{array}$

\section{Introduction}

Three-dimensional (3D) separations, which have been identified as inherent and inevitable flow features of axial compressors, can severely affect compressor performance. For example, the low momentum flow caused by separations can cause remarkable blockage in the blade passage, which will limit the loading and static pressure rise of the compressor; additionally, the mixing of the separating flow with its surrounding flows can lead to increased loss and decreased efficiency $[1,2]$. Hence, certain studies in the past few decades have discussed the importance of 3D flows in axial compressors. Several studies were also conducted to clarify the flow mechanisms and aerodynamic features of turbomachinery.

In a compressor stator passage, 3D separations mainly occur at the junction of the blade suction surface and endwall. The boundary layer on the blade suction surface has a tendency to grow thick due to adverse streamwise pressure gradient. In addition, the cross-flow pressure gradient drives the low-momentum flow of the endwall to move across the passage from the pressure surface to the suction surface of the adjacent blade. The low-momentum flow interacts with the aforementioned blade suction surface boundary layer; then, the 3D corner separation forms. According to Lei et al. [3], when the strength of corner separation is weak, i.e., no significant flow recirculation can be observed on the endwall, the resulting flow blockage and loss are very small. However, the blade loading increases as the mass flow rate of the compressor decreases. Thus, corner separation becomes stronger, and the corner stall (a stronger type of corner separation with significant recirculation flow topology on the endwall, which was defined by Lei et al. [3]) could constantly occur prior to the large-scale blade surface flow separation [4]. Large flow blockage and loss will thus be induced. Hence, compressor performance is significantly degraded.

Based on the modeling of the effects of streamwise and circumferential pressure gradients, Lei et al. [3] and Yu and Liu [4] developed models and criteria to predict the occurrence of corner stall. In their works, they 
considered the $3 \mathrm{D}$ flow effects in a $2 \mathrm{D}$ way by using a newly defined diffusion parameter, which is similar to the diffusion factor used to evaluate blade loading. Considering that near endwall flows are highly 3D, Taylor and Miller [5] found that the traverse surface pressure gradient in the spanwise direction, which is usually ignored for 2D consideration, has significant effects on corner separation/stall. To suppress the development of endwall corner separation, 3D blade designs should be tailored to manage traverse surface pressure gradient in blade passage. Finally, Taylor and Miller [5] suggested a criterion for blade lean design to avoid open corner separation, i.e., corner stall. Compared with Lei et al. [3] and Yu and Liu [4], Taylor and Miller [5] considered more real flow behaviors for compressor 3D separation flows; however, their studies were merely confined to the flow in a single stator blade row at a single design condition. According to Taylor and Miller [5], the flow behavior in a given stator blade row of a "high risk" design compressor with deteriorating performance because of strong corner stall will exhibit extreme sensitivity to blade inlet flow conditions. "High risk" or "low risk" designs are only a single-point description. A "low risk" design may become "high risk" at off-design conditions. The redesign of one blade row in a multistage compressor could also change the flows in other blade rows [6, 7]. Hence, to clarify the flow mechanics of the $3 \mathrm{D}$ endwall separations in a stator passage, the influences of the upstream and downstream blade rows and at the design and off-design conditions should also be carefully considered.

In real compressors, some important influencing factors on the endwall 3D separation cannot be ignored, such as the rotor-stator interacting flow [8,9], the upstream cavity flow [10], the clocking effects [11], and the local flow bleeding [12]. Moreover, the behaviors of the endwall 3D separation flow could vary significantly as the operating conditions change, including mass-flow rate and rotating speed [1, 13-15]. Among the varied influencing factors in multistage environments, the rotor tip clearance flow always plays the most important role in compressor blade row matching [16]. In the experimental work of Ivey et al. [17, 18], they found that increased rotor tip clearance resulted in significant changes in the performance of the downstream stator. Stator loss increased due to the increase of the rotor casing flow blockage caused by increased tip clearance. The change of loss in the stator passage was not confined to the casing endwall, but was redistributed in the entire blade span. Smith and Key [19] conducted detailed oil-flow visualization measurements in a multistage axial compressor; they found that upstream rotor tip gap size had insignificant effects on the 3D flow separations in the stator passage at nominal loading condition (design mass flow rate condition), however, it had significant 
effects on high loading conditions (small mass flow rate condition). Liu et al. [20] conducted detailed experimental investigations in a 1.5 -stage high loading axial compressor (design loading coefficient is 0.45 , which is larger than the industry used state-of-the-art compressors); the results of their study showed that a redesigned bowed stator can successfully suppress corner separation flows at the $1.75 \%$ span rotor tip gap size configuration; however, it failed as the tip gap of the upstream rotor was reduced to $1 \%$ blade span. Obviously, the abovementioned studies indicate that the sizes of the upstream rotor tip gap can considerably affect the development of the 3D separation flows in the downstream stator passage. These effects may become stronger in compressors with highly designed loading level or compressors at high loading operating condition.

The present study investigates experimentally detailed flow fields inside a 1.5-stage highly loaded low speed axial compressor. The results were analyzed by focusing on the effects of the sizes of upstream tip gap on 3D separating flows inside the downstream stator passage. The experiments were conducted in six compressor rotor tip gap size configurations. The size of the rotor tip gap varied from $0.5 \%$ to $1.75 \%$ blade span height. Pneumatic probes were used to measure flow profiles at the upstream and downstream of each blade row. Stereoscopic particle image velocimetry (SPIV) was employed to measure the instantaneous and ensemble-averaged flow fields inside the stator passage. Detailed oil-flow visualization investigations were conducted to record the complicated 3D flow structures inside the stator passage. The effects of the size of tip gap on the 3D separation flows inside the stator passage were discussed in detail based on the results. Given the significant radial flow redistribution effect in a highly loaded compressor, the size of the rotor tip gap has a close relation to the appearance of open type corner separation, i.e., corner stall. Changing the size of the rotor tip gap also changes the states of mass-flow-rate at which corner separation transformed into the corner stall. Finally, the present study found the existence of a critical rotor tip blockage state in the transformation of corner separation types at a certain rotor tip gap configuration. This finding is valuable for the study of multistage compressor matching problems at off-design conditions.

\section{Experimental setup and layout}

\subsection{Compressor facility}

The experiment was conducted on a low-speed large-scale axial compressor (LSLSAC) test facility of 
Beihang University, which is composed of a single-stage axial compressor and inlet guide vanes (IGVs), as shown in Fig. 1. A large contraction ratio bellmouth with a $1.8 \mathrm{~m}$ outer diameter lemniscate profile equipped with flow straightener is used to provide uniform and steady inlet flow. A throttle is used to alter the operating condition of the compressor. The casing out diameter of the compressor is 1 meter, and the hub diameter is 0.6 meter. At the design condition, the rotor blade loading coefficient is approximately 0.52 . The baseline rotor tip gap is $1.75 \%$ based on annulus height ( $2.30 \%$ based on blade chord length). To investigate the effect of the rotor tip gap on the downstream flows, the gap can be reduced to $1.50 \%, 1.25 \%, 1.00 \%, 0.75 \%$, and $0.50 \%$ based on annulus height. In the following analysis, these six compressor configurations will be denoted as $1.75 \tau, 1.50 \tau$, $1.25 \tau, 1.00 \tau, 0.75 \tau$, and $0.50 \tau$, respectively. More detailed design parameters are summarized in Table 1 , which were also introduced by Du and Yu [21].

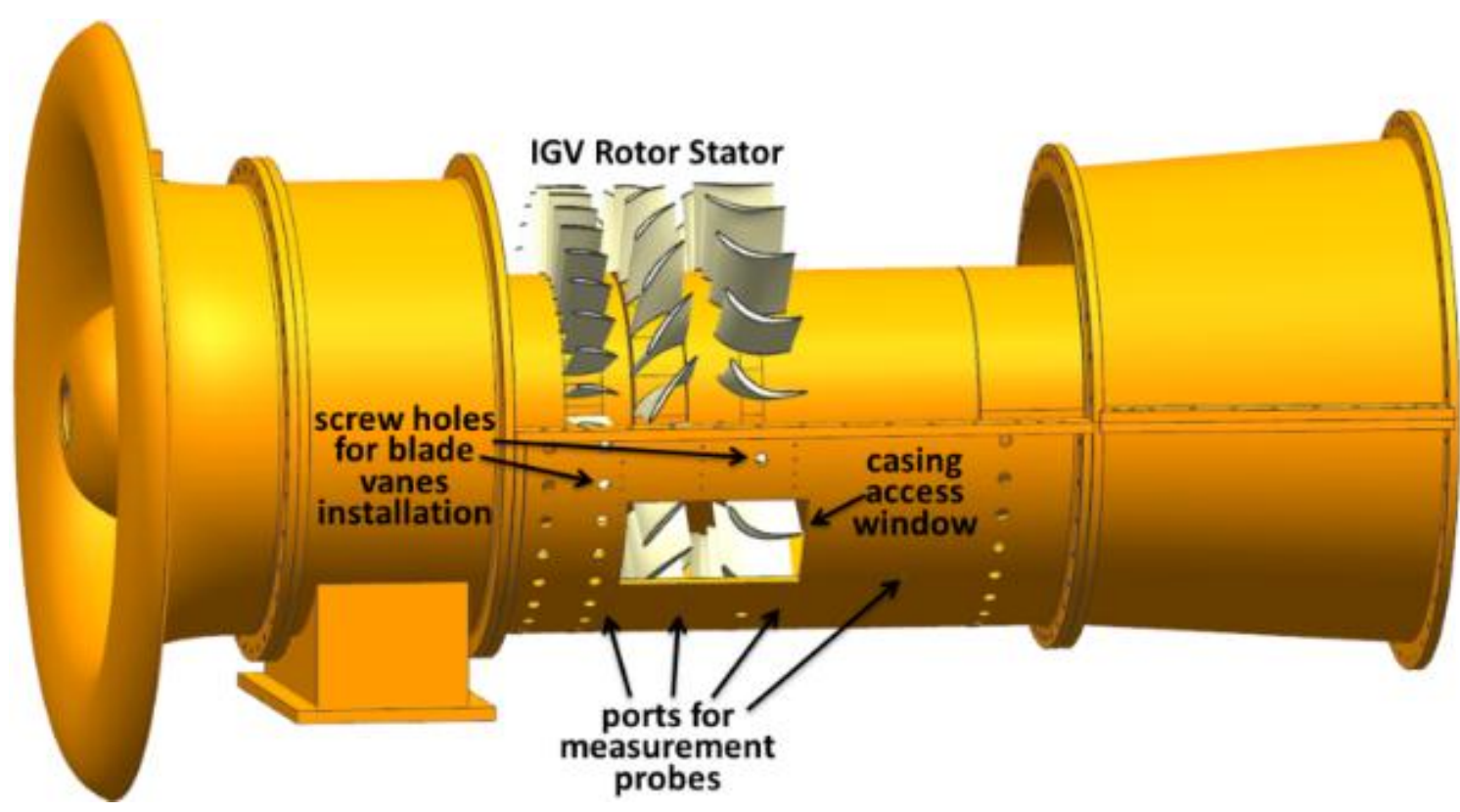

Fig. 1 Schematic plot of the low-speed large-scale compressor test facility.

Table 1 Design parameters of the compressor test facility.

\begin{tabular}{ll}
\hline \hline Outer diameter $(\mathrm{m})$ & 1.0 \\
Hub-to-tip ratio & 0.6 \\
Design speed $(\mathrm{rpm})$ & 1200 \\
Number of blades & $36(\mathrm{IGV}) ; 17$ (Rotor); 20(Stator) \\
Blade camber angle $\left({ }^{\circ}\right)$ & $7(\mathrm{IGV}) ; 40.8$ (Rotor); 42.6 (Stator) \\
Blade stagger angle $\left({ }^{\circ}\right)$ & $5(\mathrm{IGV}) ; 36.5$ (Rotor); 14.7 (Stator) \\
Blade height $(\mathrm{m})$ & $0.2(\mathrm{IGV}) ; 0.2$ (Stator) \\
Blade chord $(\mathrm{m})$ & $0.095(\mathrm{IGV}) ; 0.152$ (Rotor); 0.18(Stator) \\
Rotor tip gap $(\mathrm{mm})$ & $1.0 / 1.5 / 2.0 / 2.5 / 3.0 / 3.5$ \\
\hline \hline
\end{tabular}




\subsection{Pneumatic probe measurements}

The pneumatic probe measurement locations of the compressor facility are shown in Fig. 2. At Plane 0, four circumferential static pressure taps mounted in the casing is used to measure mass flow coefficient. Four circumferential static pressure taps mounted in the casing at Plane 5 and six circumferential static pressure taps mounted in the casing at Plane 1 are used to measure the static pressure rise of the compressor. A five-hole probe is used to measure 3D velocity and pressure profiles at the outlet of the rotor and stator blade rows, i.e., at Plane 3 and Plane 4 in Fig. 2. The detailed arrangement of the five-hole probe's measurement points is shown in [22]. The measurement uncertainties of the flow angles are less than 1 degree. The uncertainty of the measured total pressure is less than $0.8 \%$. The uncertainties of the measured velocities are less than $2.0 \%$.

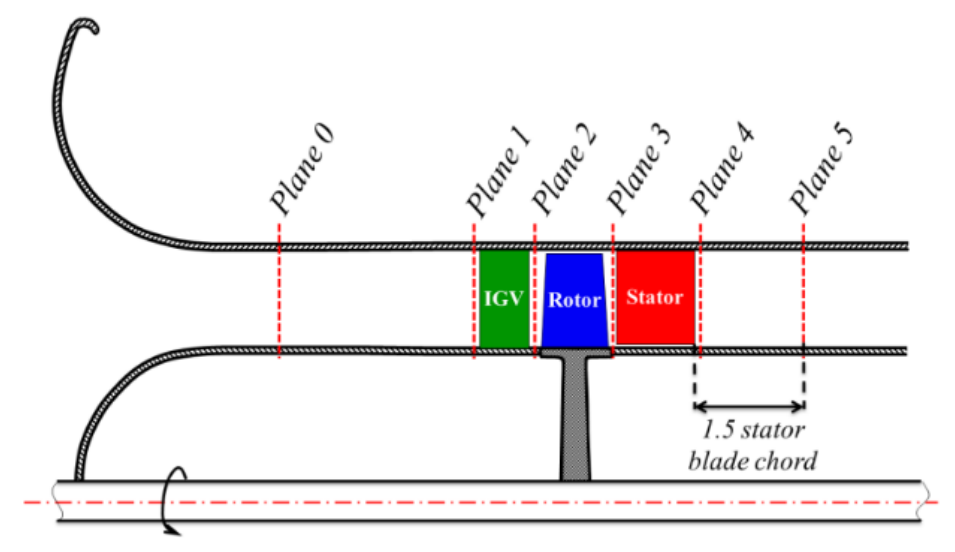

Fig. 2 Measurement location of the pneumatic probe.

\subsection{SPIV measurements}

The five-hole probe is used to measure the flow between blade rows, but it not suitable for measuring the flow inside the blade passage. In the present study, a commercial SPIV system, developed by LaVision Incorporation, is employed to measure the velocity field inside the stator passage. Detailed parameters on the SPIV system can be found in [21]. Three measurement cross-sections, i.e., 40\%, 63\%, and 84\% blade chord length away from the blade leading edge are chosen as shown in Fig. 3. Each measurement cross-section is nearly perpendicular to the lateral suction surfaces at the blade tip. Given the combined effects of geometric restriction of the blade passage and strong flare light at the hub surface, the final effective measured field for every cross-section is about $140 \mathrm{~mm}$ (in the spanwise direction) $\times 100 \mathrm{~mm}$ (in the pitchwise direction). Detailed experimental setup and data processing methods were introduced by Zhang [23]. The uncertainty of the 
ensemble-averaged velocity is adequately accurate with an uncertainty of about $1.0 \%, 2.5 \%$ and $1.4 \%$ in the mainstream region, in the tip region and at the region below $60 \%$ blade span, respectively [24].

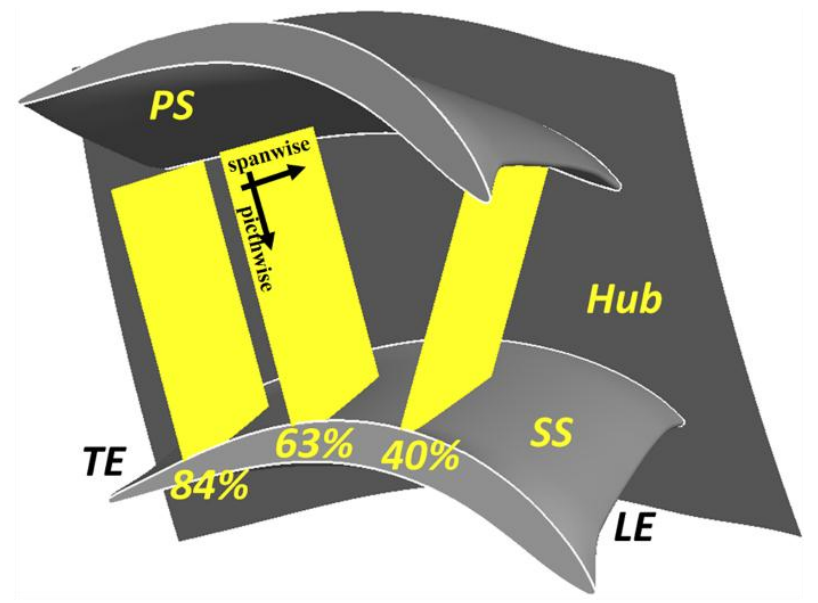

Fig. 3 Schematic layout of the measurement cross sections.

\subsection{Oil-flow visualization}

In the present experiments, oil-flow visualization tests are conducted to reveal qualitatively the flow patterns on the endwall and blade surfaces of the stator passage. Discussions will focus on the corner separations that form at the both the hub and tip endwall junctions on blade suction surface. As shown in Fig. 4, two measurement materials are used to make the skin-friction lines appear clearer: the white material, which is a mixture of industrial silicone oil and titanium dioxide, and the red material, which is a mixture of industrial silicone oil and solid power. The runtime of each test was between 5 and 10 minutes. To access the repeatability of the oil-flow visualization measurement, repetitive experiments are conducted at different dates. The repetitive results at high flow rate are shown in Fig. 4a and Fig. 4b, and the repetitive results at low flow rate are shown in Fig. $4 \mathrm{c}$ and Fig. 4d. It can be seen that the applied oil-flow visualization is stable and reliable.

\section{Overall characteristics of the compressor}

Generally, compressor performance will deteriorate with the increase of rotor tip clearance. For example, Smith [25] found that $1 \%$ increase of the rotor tip clearance could result in $4.6 \%$ decrease of pressure rise; Baghdadi [26] found that $1 \%$ increase of the rotor tip clearance would bring about $8 \%$ decrease of the steady margin. Compressor characteristics with six different rotor tip gaps are shown in Fig. 5, in which the characteristics are expressed as static pressure rise coefficient, which is a function of mass flow coefficient. It 
can be seen that compressor static pressure rise decreases with the growth of the rotor tip gap (from $0.50 \tau$ to $1.75 \tau)$. At high flow rates, where $\varphi>0.56$, the difference of the static pressure rise between two adjacent rotor tip gap configurations is very small. Thus, at some conditions distinguishing characteristic from each other in the performance map is difficult. At low flow rates, where $\varphi<0.56$, the difference of the characteristics between the six configurations is significant. In addition, the steady operation range decreases with the increase of the rotor tip gap.
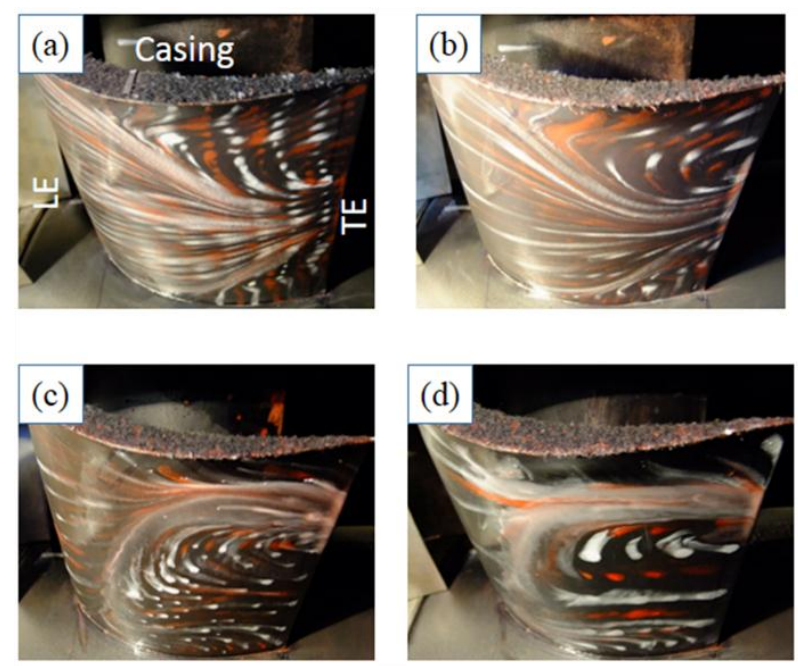

Fig. 4 Repeatability test at high flow rate $(a, b)$ and low flow rate $(c, d)$.

(a)

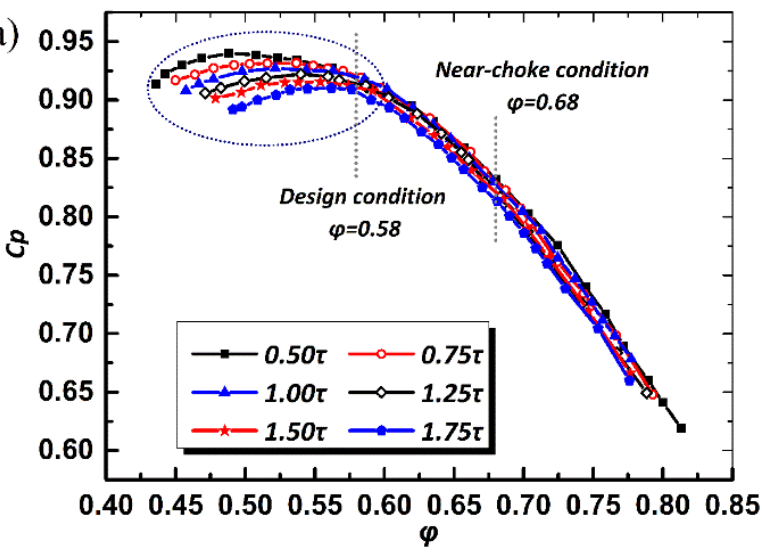

(b)

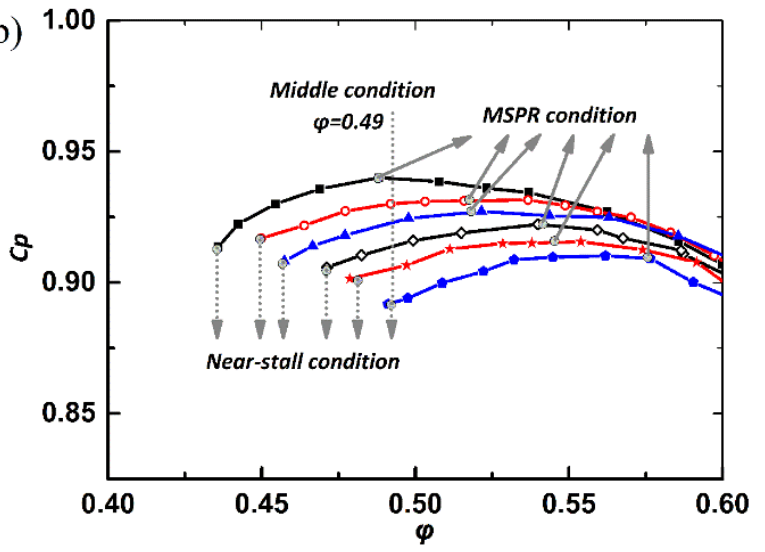

Fig. 5 Characteristics of the tested compressor: (a) the whole operating lines; (b) the amplification of part of the operating lines that surrounded by the blue dash ellipse in (a).

\section{General 3D flow structures inside the stator passage}

\subsection{Oil-flow visualization results}

For every rotor tip gap compressor configuration, oil-flow visualization is conducted at five flow rate 
conditions (except for $0.50 \tau$ ): near-choke condition (NC, $\varphi=0.68$ ), design condition (DE, $\varphi=0.58$ ), maximum static pressure rise condition (MSPR, the flow rate of this condition depends on the compressor characteristics), middle condition (MID, $\varphi=0.49$ ), and near-stall condition (NS, the flow rate of this condition also depends on the compressor characteristics). These conditions are depicted on the performance map in Fig. 5. The detailed mass flow coefficient of these operating conditions is shown in Table 2.

Table 2 Mass flow coefficient of the operating conditions for the oil-flow visualization measurements.

\begin{tabular}{lllllll}
\hline \hline & $0.50 \tau$ & $0.75 \tau$ & $1.00 \tau$ & $1.25 \tau$ & $1.50 \tau$ & $1.75 \tau$ \\
\hline \hline NC & 0.68 & 0.68 & 0.68 & 0.68 & 0.68 & 0.68 \\
DE & 0.58 & 0.58 & 0.58 & 0.58 & 0.58 & 0.58 \\
MSPR & 0.485 & 0.52 & 0.52 & 0.54 & 0.545 & 0.555 \\
MID & & 0.49 & 0.49 & 0.49 & 0.49 & \\
NS & 0.438 & 0.45 & 0.458 & 0.47 & 0.478 & 0.485 \\
\hline \hline
\end{tabular}

In the present study, oil-flow visualization is conducted on the blade suction surface, blade pressure surface, and the endwalls of the stator passage. The oil-flow results on the blade suction surface of all operating conditions are shown in Fig. 6. The 3D separating flow at the NS condition of $1.75 \tau$ is extremely unstable, and the present result is the most common situation. The other two possible results are shown in Fig. 13. If there is no special instruction in the following analysis, the present result in Fig. 6 will be regarded as the result of the NS condition. From Fig. 6 it can be seen that in most of the operating conditions only two separation lines are on the blade suction surface, which are denoted as $S L 1$ and $S L 2$. Separation line $S L 1$, which originates from the juncture of the blade suction surface and the casing and ends as a focus point, corresponds to the tip corner separation. Separation line $S L 2$, which originates from the juncture of the blade suction surface and the hub and also ends as a focus point, corresponds to hub corner separation. These two corner separations comprise the main 3D flow structures inside the stator passage.

\subsection{Representative flow topologies along the compressor operating line}

As shown in Fig. 6, the separation scale is different at different operating conditions. Based on the development of corner separation, the 3D flow structures inside the stator passage can be classified into four types, which are shown in Fig. 7. The schematic streamlines are drawn based on the oil-flow results, separation/attachment lines, and critical points are obtained according to the topological theory of the 3D steady 
flow patterns introduced by Perry et al. [27] and Surana et al. [28, 29]. SPIV measurements are only conducted at the NC/DE/NS conditions of the $1.00 \tau$ and the NC/DE/MID conditions of $1.75 \tau$. Thus, only the results for these two configurations are accompanied with the velocity field inside the stator passage.
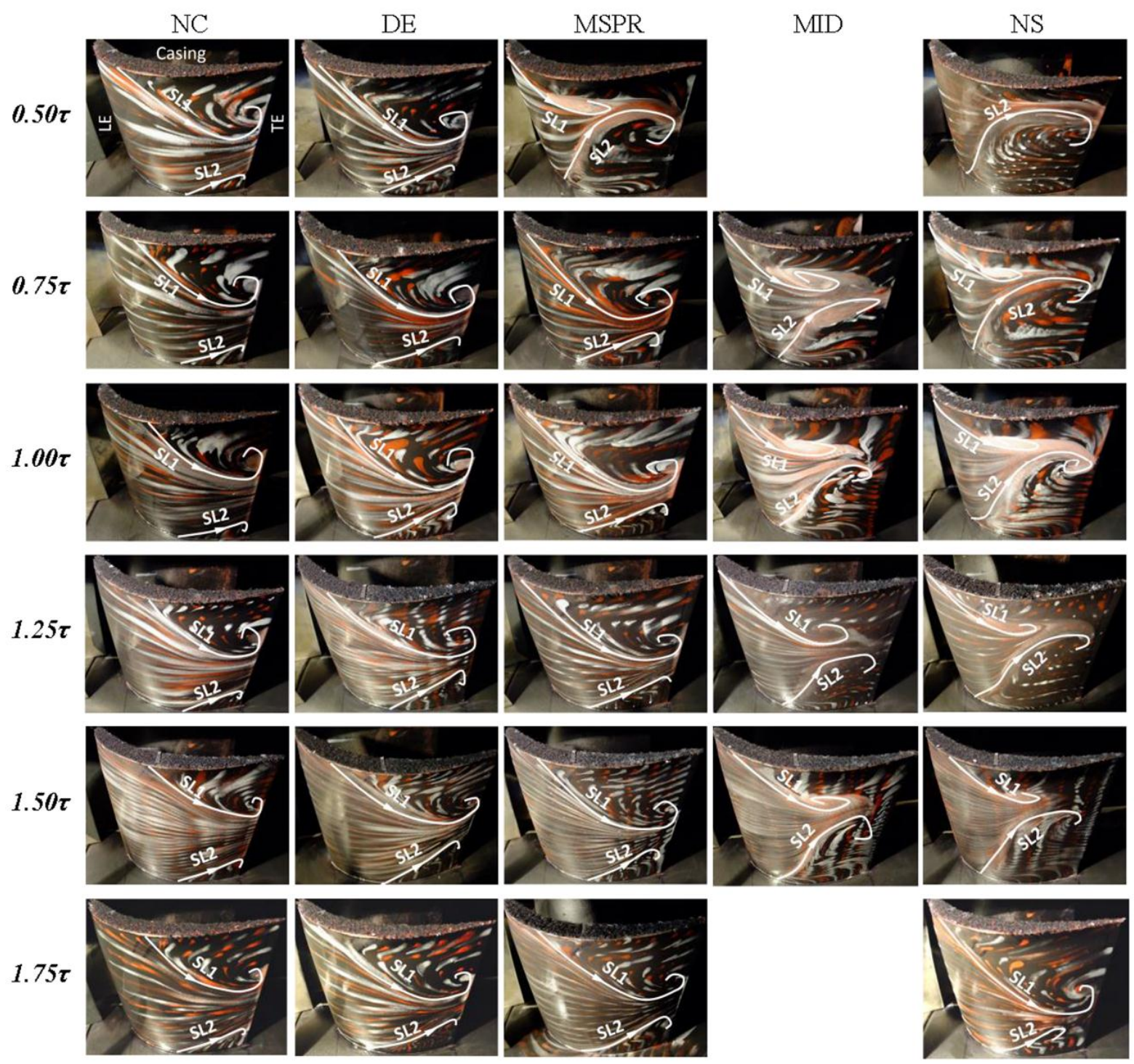

Fig. 6 Oil-flow results on the blade suction surface of all the operating conditions.

The first typical 3D flow structure inside the stator passage, which is denoted as Type A, is shown in Fig. 7a.

This structure can represent the results at high flow rate conditions for all the six configurations. Separation line $S L 2$ on the blade suction surface and separation line $S L 3$ on the hub comprise the hub corner separation, whereas separation line $S L 1$ on the blade suction surface and another separation line on the casing (not shown here) comprise the tip corner separation. The tip corner separation is bigger than the hub corner separation, but both of them are very small and the induced flow blockage is not prominent. 
The second typical 3D flow structure inside the stator passage, which is denoted as Type B, is shown in Fig.

7b. This structure is the next phase of Type $A$ as the flow rate decreases. Compared with Type A, both the tip corner separation and hub corner separation (especially for the tip corner separation) become bigger, and the main characteristic lines and critical points do not change (except for the appearance of saddle point $S 1$, which is induced by the interaction of tip corner separation and hub corner separation). The resulting blockage for this type near the trailing edge occupies the entire span, but only covers a very small area in the pitchwise direction, as shown in the SPIV results of Fig. $7 b$.

(a) Type A

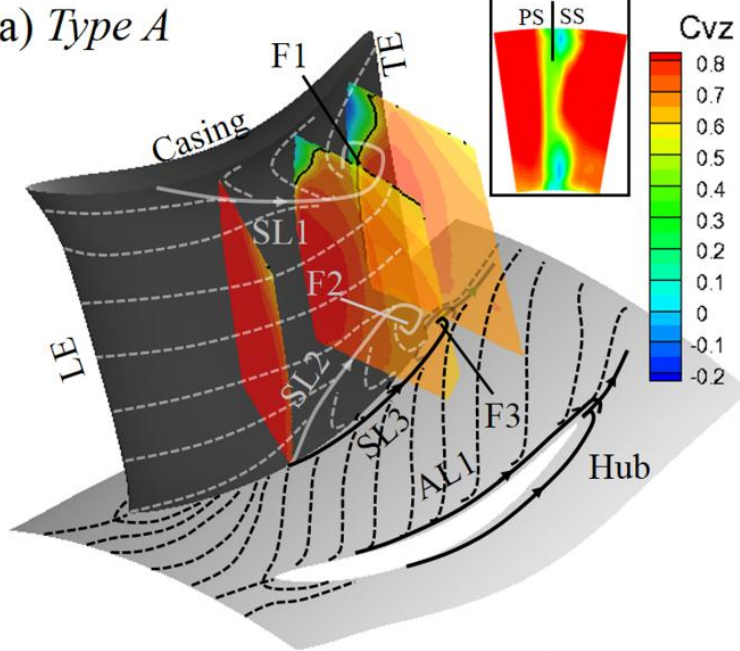

(c) Type $C$

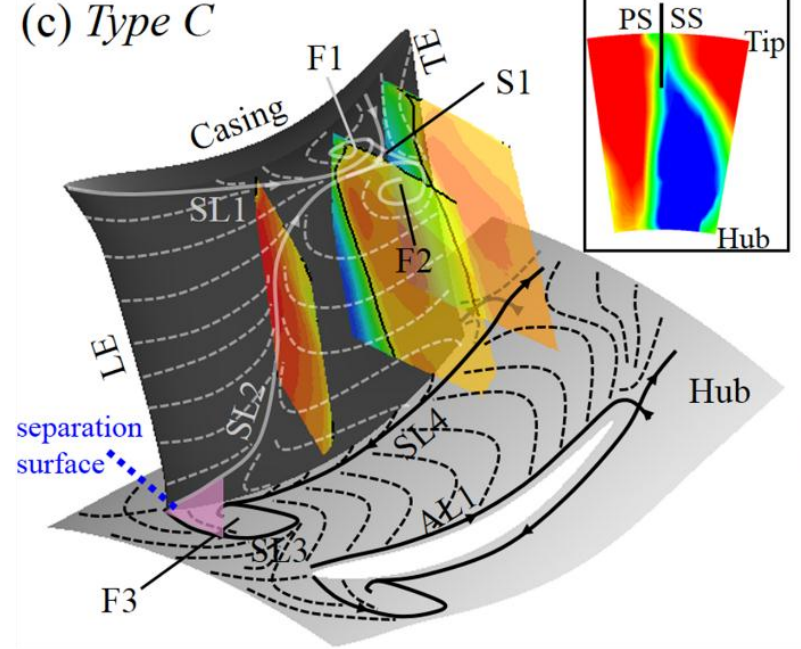

(b) Type B

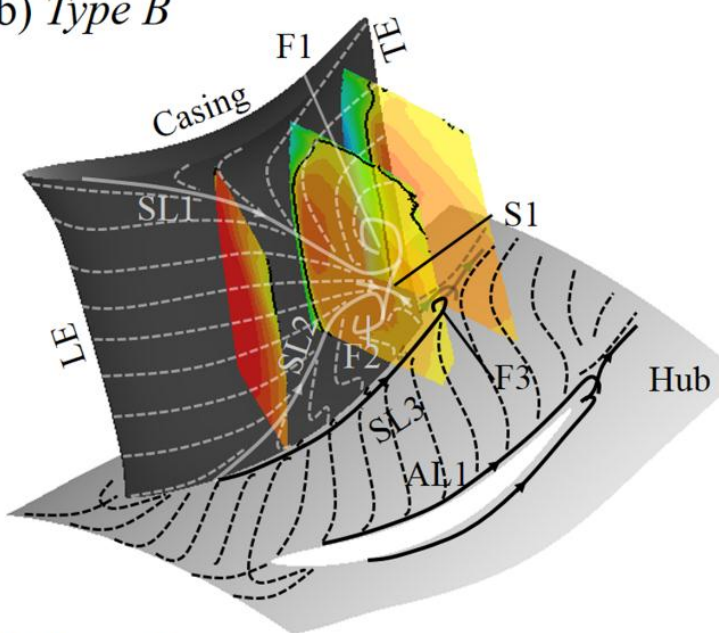

(d) Type D

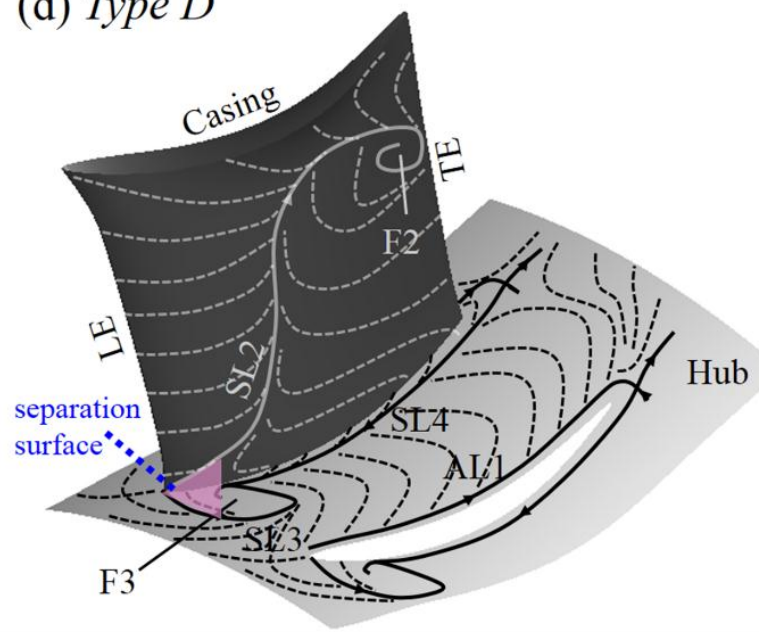

Fig. 7 Typical topological structures of the separation flow in the stator passage. (Dash lines denote the limiting streamlines; solid lines along with arrows denote the separation lines/attachment lines; contours denote the streamwise velocity measured using the SPIV; the dark lines in the contours denote the edge of the blockage region.)

The third typical 3D flow structure inside the stator passage, which is denoted as Type $C$, is shown in Fig.

7c. This structure can represent the 3D flow structures at low flow rate conditions for most of the configurations. 
The tip corner separation for Type A and Type B is bigger than the hub corner separation. However, the hub corner separation of Type $C$ is bigger than the tip corner separation. This bigger hub corner separation results in a significant blockage not only in the spanwise direction, but also in the pitchwise direction at the hub corner. This type of corner separation, which is known as open corner separation, was investigated by Taylor and Miller [5]. As shown in Fig. 7c, separation line SL3, which originates from the blade leading edge along with separation line $S L 2$, composes a separation surface. This feature distinguishes between closed corner separation (hub corner separation in Type A and Type B) and the open type. The sudden increase of hub corner separation prompts most of the fluid inside the stator passage flow through the tip region, which improves the flow condition and reduces the tip corner separation.

The last typical 3D flow structure inside the stator passage, which is denoted as Type D, is shown in Fig. 7d. This structure is only observed at the NS condition of the $0.50 \tau$ configuration. Compared with Type C, hub corner separation becomes stronger and induces a bigger blockage at the bottom of the blade passage. Thus, much more fluid flow through the tip region and the tip corner separation disappears.

\section{Effects of the size of rotor tip gap on the evolution of flow topologies}

Rotor tip leakage flow plays an important role not only in efficiency and pressure rise but also on the stability of the operating range of a compressor. The effect of rotor tip gap on the flow of the following stator passage is mainly reflected in the redistribution of inlet flow along the spanwise direction. In early 1985, Wisler [7] found that increased rotor tip gap could generate a larger tip leakage vortex resulting in additional blockage near the tip endwall at the inlet of the following stator. Zhang [23] studied the rotor tip leakage flow of the investigated compressor, and found that the leakage vortex is enhanced and the induced flow blockage is enlarged with the increase of the rotor tip gap tip. At the same flow rate condition, enlarged blockage drives more fluid pass through the lower half of the stator span. This mechanism locally increases mass flow rate and unloads the hub (as shown in Fig. 8), which results in reduced hub corner separations. By contrast, enlarged blockage decreases mass flow rate and increases loading at the tip region (as shown in Fig. 8); these two results lead to enhanced tip corner separations. 


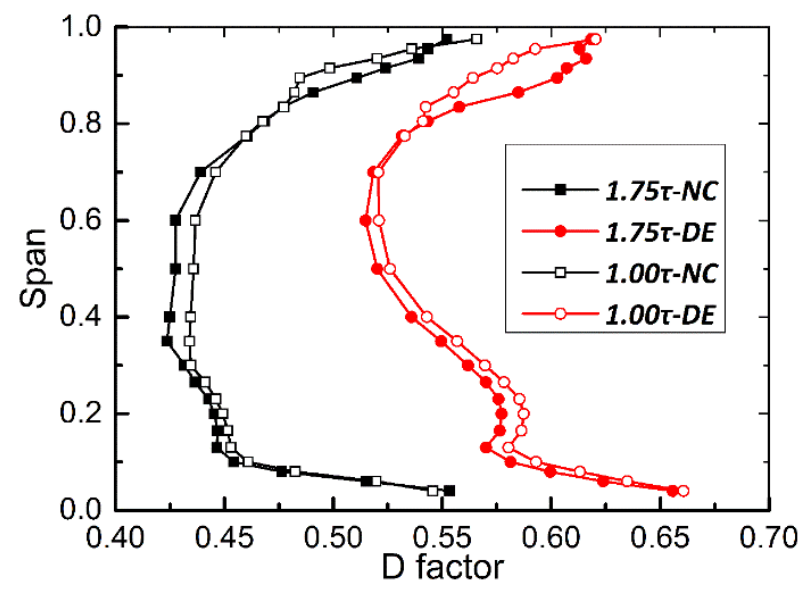

Fig. 8 The diffusion factor of the stator passage.

\subsection{Variation of separation scale}

At the edge of blade trailing, the scale of corner separation in the spanwise direction could be set as a metric to evaluate the strength of the 3D separation. The metric in the oil-flow results shown in Fig. 6 is extracted and plotted versus the mass flow coefficient, as shown in Fig. 9. The three possible results at the NS condition of $1.75 \tau$ are all plotted here. For the $0.50 \tau$ configuration, the tip corner separation at NC condition covers $35 \%$ blade height, whereas the hub corner separation covers $25 \%$ blade height. Tip corner separation increases to $40 \%$ blade height as flow rate decreases, and hub corner separation increases to $35 \%$ blade height at DE condition. As flow rate decreases sequentially, the hub corner separation persistently increases, i.e., it increases to $75 \%$ blade height at MSPR condition and $82.5 \%$ blade height at NS condition. Tip corner separation decreases to $25 \%$ blade height at MSPR condition and totally disappears at NS condition. For the other five compressor configurations, namely, $0.75 \tau, 1.00 \tau, 1.25 \tau, 1.50 \tau$, and $1.75 \tau$, the evolutionary tendency of the corner separation is similar to that of the $0.50 \tau$ configuration. That is, hub corner separation increases monotonously with the decrease of flow rate while tip corner separation increases initially before decreasing at the last one/two flow rate conditions. However, the separation scale in different rotor tip gap configurations is different at the same flow rate condition.

The difference of the separation scale between different rotor tip gap configurations is very small at high flow rate conditions (such as $\mathrm{NC}$ and $\mathrm{DE}$ ). Assessing the effect of rotor tip gap size on corner separation from the oil-flow results is difficult. However, the five-hole probe results (as shown in Fig. 10) clearly shows that as the rotor tip gap increases from $1.0 \%$ to $1.75 \%$, mass flow rate in the tip region is reduced. By contrast, mass 
flow rate in the near hub region is increased, which is induced by enlarged tip corner separation and decreased hub corner separation.

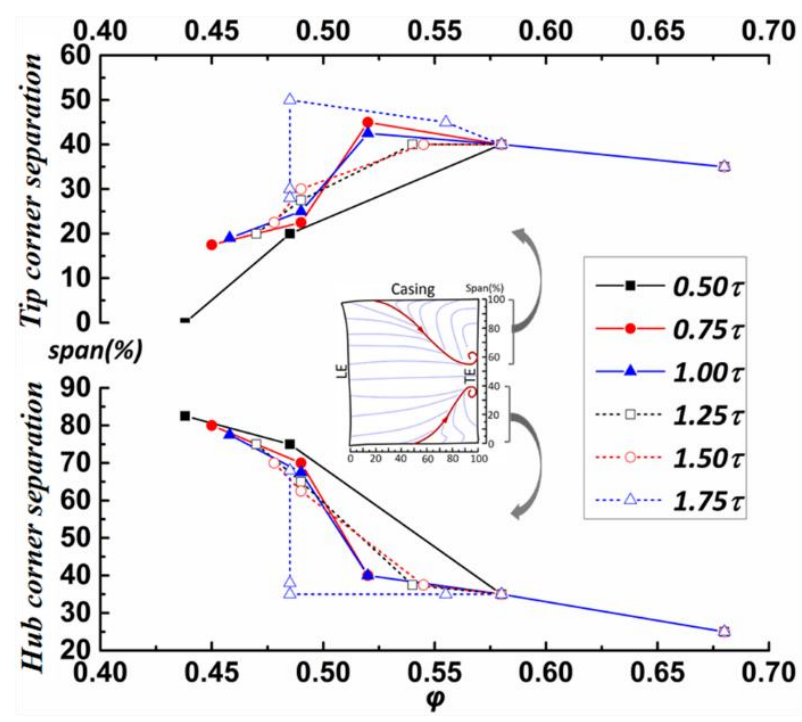

Fig. 9 Variation of the corner separation scale with the mass flow rate.

The difference of the separation scale between different rotor tip gap configurations is significant at low flow rate conditions. To assess the effect of rotor tip gap size, the separation scale at the MID condition (the MSPR condition for $0.50 \tau$ and the NS condition for $1.75 \tau$ ) of different rotor tip gap configurations is extracted from Fig. 9, as shown in Fig. 11. Tip corner separation scale is enlarged and the hub corner separation scale is reduced as the rotor tip gap increases. Before the rotor tip gap reaches $1.75 \tau$, the variation of the corner separation scale is almost linear with the increase of rotor tip gap.

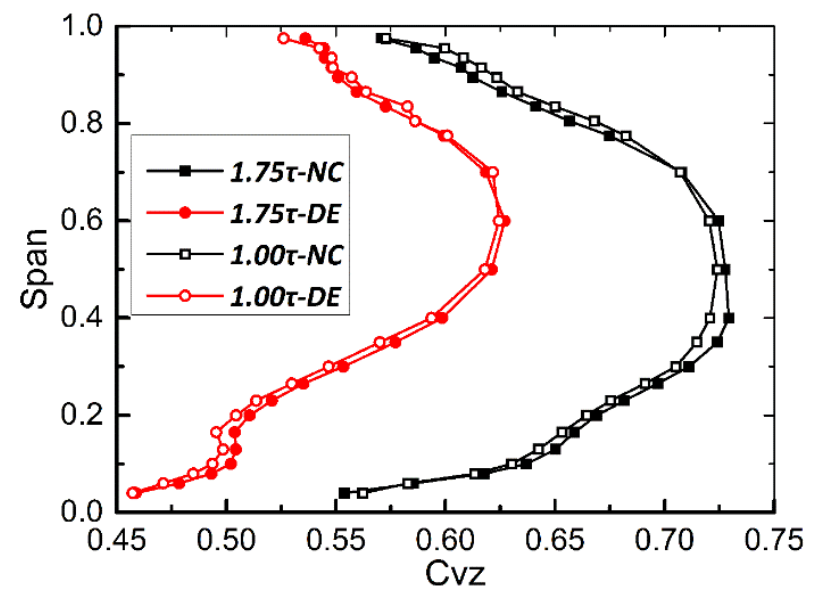

Fig. 10 The circumferential averaged distribution of the axial velocity at the outlet of the stator.

The separating flow at the NS condition is very unstable for $1.75 \tau$ because three separating flow structures are possible in this condition. Although the result presented in Fig. 6 is the most common situation, the 
separating flow structure in the five-hole probe results measured at a lower mass flow rate condition $(\varphi=0.48)$ at the stator outlet (not shown here) is featured as bigger hub corner separation with smaller tip corner separation. Hence, fast variation of the separation scale exists around the NS condition. Hub corner separation increases and the tip corner separation decreases, which could be the result of the transformation from closed hub corner separation to open hub corner separation. Thus, the 3D flow structure inside the stator passage is featured as Type B before this variation. After this variation, the 3D flow structure inside the stator passage is featured as Type C.

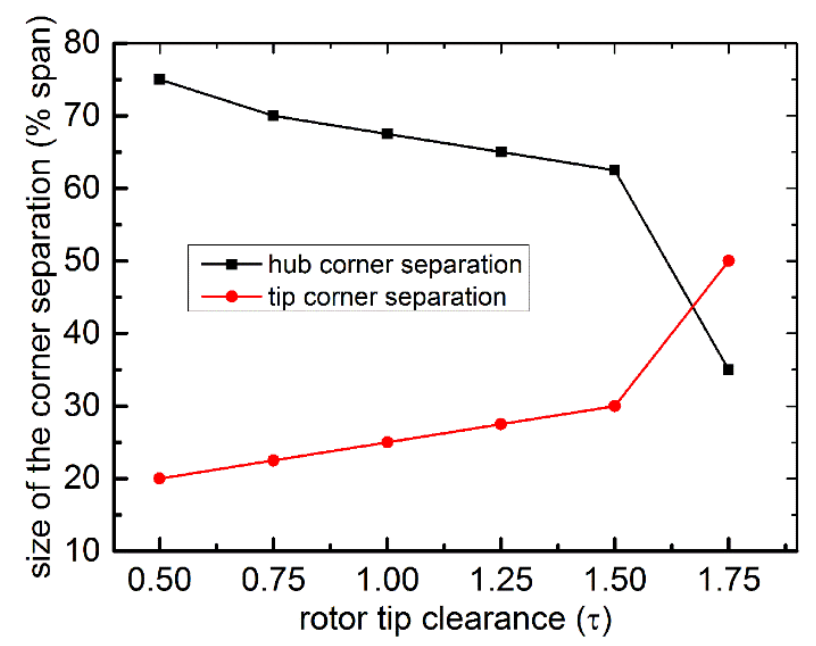

Fig. 11 Variation of the corner separation scale with the rotor tip gap (at the MID condition).

\subsection{Transformation of the hub corner separation from closed separation to open type}

The transformation of hub corner separation from closed separation to open type was studied by Taylor and Miller [5]. They proposed a metric for the magnitude of the streamline curvature to determine whether corner separation will become open separation. The following analysis shows that the transformation of the hub corner separation from closed separation to open type happens at a very short stage on the compressor operating line. Around this short stage, the 3D separating flow inside the stator passage is very unstable.

The SPIV results of the $84 \%$ chord cross-section at the NS condition of the $1.75 \tau$ configuration is shown in Fig. 12. In the averaged flow field, the width of the flow blockage region in the pitchwise direction is almost the same along the span. Actually, three typical flow fields exist in the instantaneous results: flow blockage occurs at the lower half part of the passage (see Fig. 12b); flow blockage occurs at the higher half part of the passage (see Fig. 12c); and flow blockage occurs at the whole passage (see Fig. 12d). From Fig. 7 it can be seen that the 
flow field in Fig. 12b corresponds to the typical 3D flow structure Type $C$. This result means that at the NS condition the hub corner separation is already open type at some moments. However, because that in the instantaneous results the proportion of the flow structure shown in Fig. $12 \mathrm{~b}$ is very small, the flow field in the averaged results is featured as Type B. Oil-flow visualization is repeated several times at the NS condition. The present oil-flow result in Fig. 6 is found to be the common situation. The other two flow structures shown in Fig. 13b and Fig. 13c (the flow structure in this figure corresponds to Type C) will also occur occasionally. Hence, the transformation of hub corner separation for $1.75 \tau$ configuration from closed separation to open type should happen around this mass flow rate condition, but will only last for a short stage. Before this stage, the 3D flow structure inside the stator passage is stable and is featured as Type B. After this stage, the 3D flow structure inside the stator passage is also stable and is featured as Type C. However, right during this stage the flow is very unstable and any small perturbation may prompt the transformation from Type B to Type $C$.

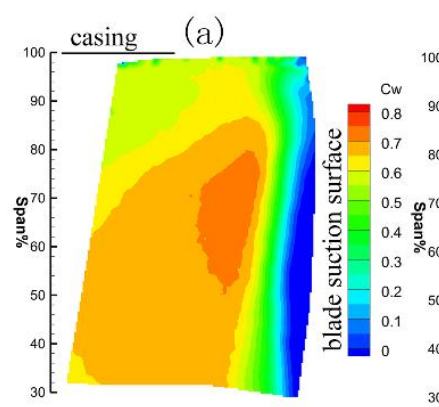

(b)

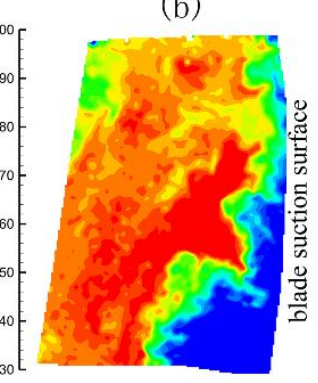

(c)

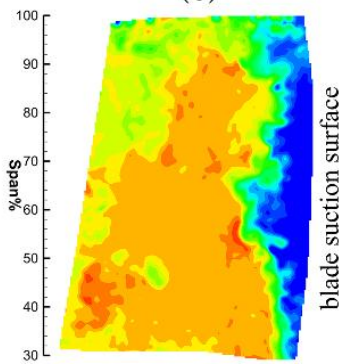

(d)

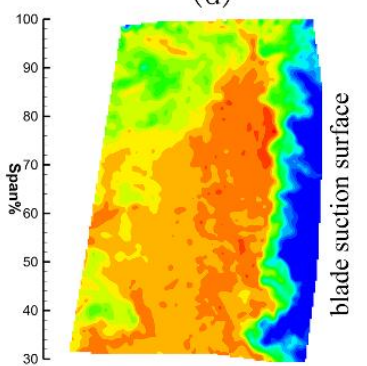

Fig. 12 The streamwise velocity at the $84 \%$ chord cross section at the NS condition of the $1.75 \tau$ configuration: (a) the averaged results; $($ b) $/(\mathbf{c}) /(d)$ : the instantaneous results.
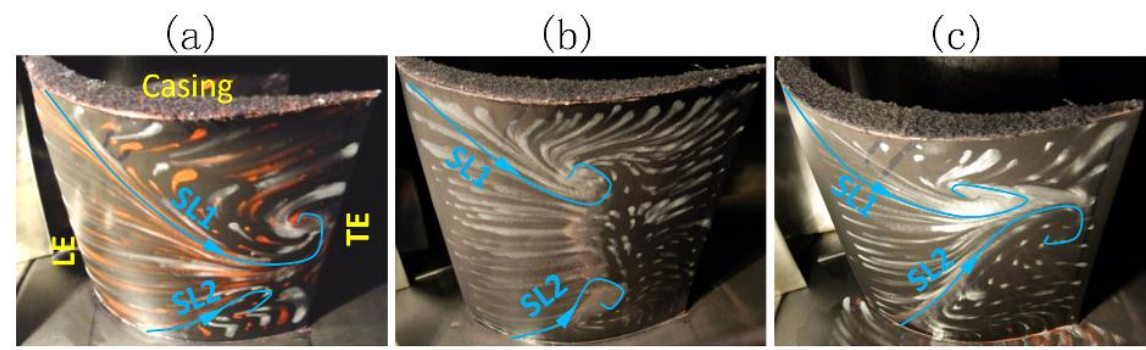

Fig. 13 Three possible oil-flow results around the NS condition of the $1.75 \tau$ configuration.

\subsection{Variation of flow topology types}

For $1.75 \tau$ configuration, the transformation of hub corner separation happens around the NS condition, where $\varphi=0.485$. The unstable stage on the compressor operating line should also exist for the other five compressor configurations. However, because oil-flow visualization was only conducted at four/five discrete conditions, the accurate flow rate of this unstable stage on the compressor operating line cannot be obtained 
directly.

The development of the 3D separating flow inside the stator passage is closely associated with the flow incidence of the stator, wherein a critical flow incidence exists, which can turn the closed corner separation into open type $[3,5]$. For the $1.75 \tau$ and $1.00 \tau$ configurations, the circumferential averaged distribution of the stator inlet flow angle is shown in Fig. 14a. It should be noted that for the 5-hole probe measurement the mass flow rate coefficient at the NS condition is 0.48 and 0.45 for $1.75 \tau$ and $1.00 \tau$, respectively. To compare the results at the same small mass flow rate condition, the inlet flow angle at $\varphi=0.48$ of the $1.00 \tau$ configuration is obtained using the interpolation from the results of NC, DE, and NS conditions, as shown in Fig. 14a. At the same mass flow rate condition, the variation of the stator inlet flow angle with the rotor tip gap size (from $1.00 \tau$ to $1.75 \tau$ ) is shown in Fig. 14b. As the rotor tip gap size increases (from $1.00 \tau$ to $1.75 \tau$ ), because the increased rotor tip blockage prompts more fluid to flow through the lower part of the passage, the stator inlet flow angle in the rotor tip region is enlarged, and the stator inlet flow angle near the blockage region and near the hub is reduced. In addition, the variation of stator inlet flow angle with the rotor tip gap size increases as mass flow rate decreases. In modern compressors, blades will diffuse the flow efficiently over the middle $60 \%$ to $80 \%$ of the span, thus, the endwall and corner regions are key to aerodynamic blockage, loss production, and compressor stability [3]. Hence, the reduced stator inlet flow angle near the hub has a very important influence on the development of corner separation. Here, the endwall regions are defined as the $0 \%$ to $20 \%$ of span near the hub. The mass-averaged stator inlet flow angle in this region is shown in Fig. 15a. The variation of the averaged inlet flow angle with the mass flow rate is almost linear. Thus, this variation could be fitted using a linear function, and the results of $1.50 \tau, 1.25 \tau, 0.75 \tau$ and $0.50 \tau$ configurations could be obtained through linear interpolation, as shown in Fig. 15b. For $1.75 \tau$ configuration, the transformation of hub corner separation happens around $\varphi=0.485$. Thus, it can be deduced that the critical inlet flow angle for this stator is $47.3^{\circ}$. Hence, the mass flow coefficients where the transformation of the hub corner separation happens for $1.50 \tau, 1.25 \tau, 1.00 \tau, 0.75 \tau$, and $0.50 \tau$ configurations are $0.494,0.501,0.508,0.514$, and 0.520 , respectively.

The above analysis shows that, as flow rate decreases, the 3D flow structures inside the stator passage are featured as Type A, Type B, Type C, and Type D in sequence. An unstable stage exists between Type B and Type $C$. This unstable stage will occur earlier when the rotor tip gap decreases. Hence, the distribution of the typical 3D flow structures along the compressor operating line for different compressor configurations can be drawn as 
Fig. 16. The blue arrows in this figure denote the oil-flow visualization operating conditions. For the $1.75 \tau$ configuration, this unstable stage occurs around the NS condition. However, for other configurations it occurs before the MID condition. Thus, a sharp increase (decrease) can be seen in the tip (hub) corner separation from $1.50 \tau$ to $1.75 \tau$ in Fig. 11.
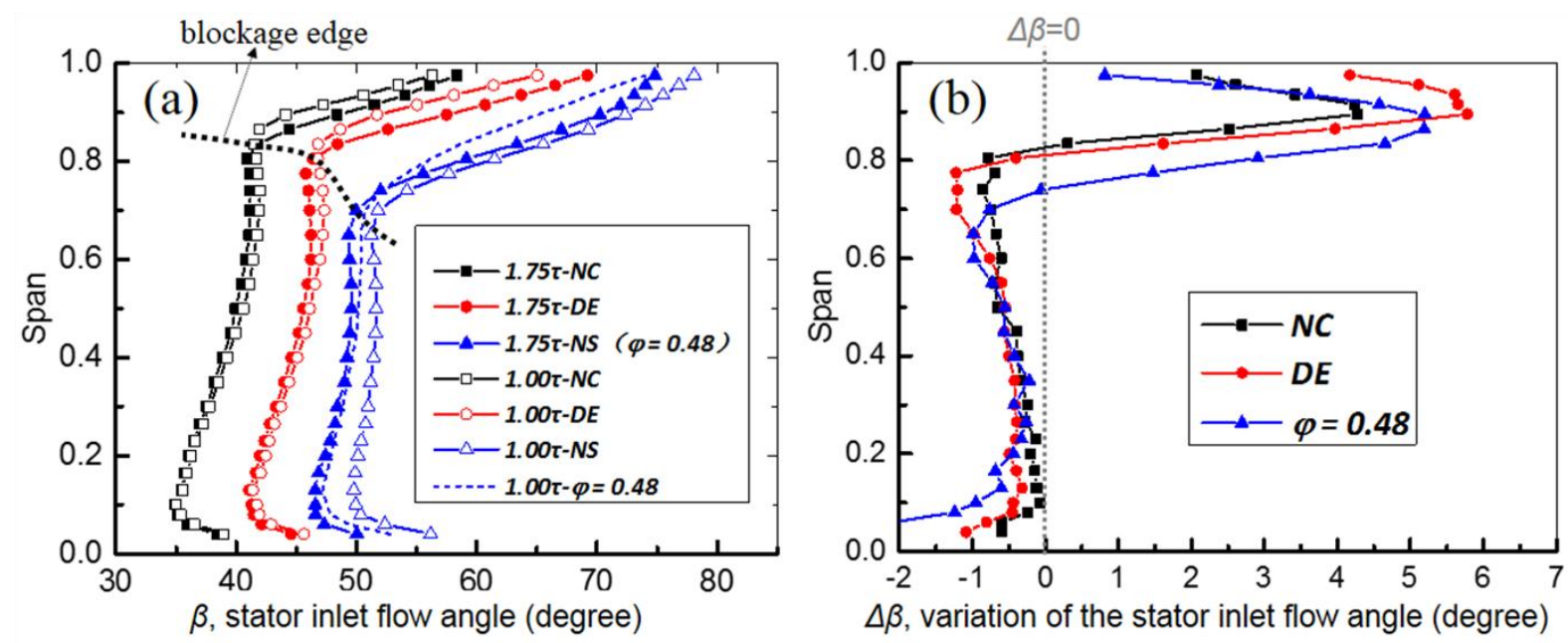

Fig. 14 The circumferential averaged distribution of the inlet flow angle of the stator.
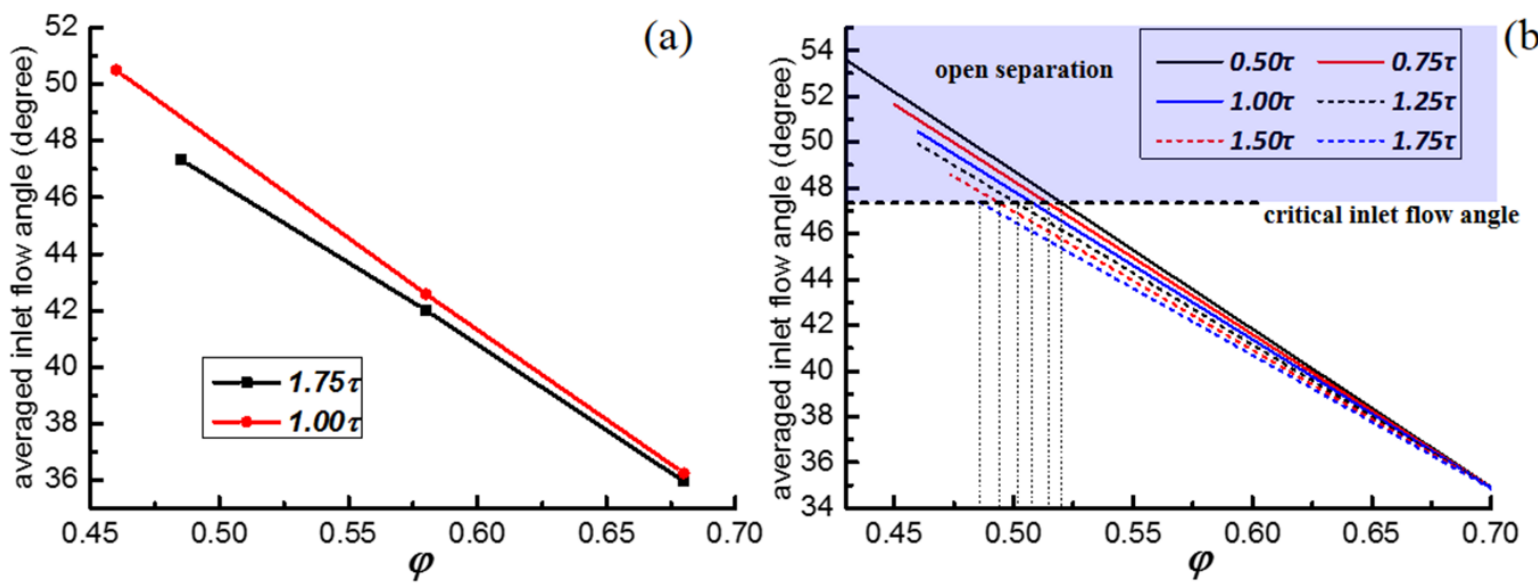

(b)

Fig. 15 Variation of the averaged inlet flow angle with the mass flow rate.

\subsection{Quantitative analysis of the effect of the size of rotor tip gap on 3D separating flows}

As mentioned before, the unstable stage on the compressor operating line varies with the change of the size of rotor tip gap. The direct effect of rotor tip gap is the induced rotor tip blockage, and this tip blockage will redistribute the mass flow rate along the blade height. At a given mass flow rate condition (around the unstable stage), the flow incidence at the hub region increases as the rotor tip blockage decreases. Hence, based on the 
aforementioned critical flow incidence, a critical rotor tip blockage should exist.

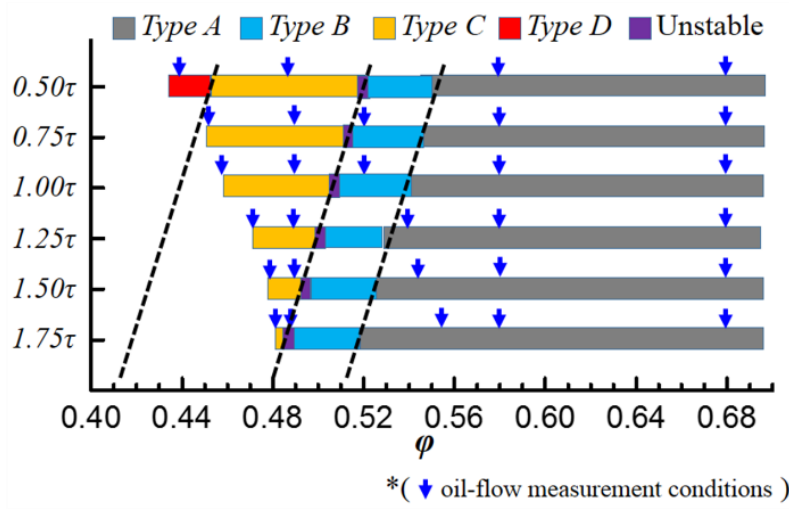

Fig. 16 Variation of flow topology types with the mass flow rate.

The axial velocity fields at the rotor outlet obtained using the five-hole probe is shown in Fig. 17. A one-dimensional parameter, which is defined by Yu et al. [30], is used to assess the rotor tip blockage effect,

$$
B_{m}=\frac{m_{b}}{m_{t}}=\frac{\rho \int_{A_{b}}(\bar{W}-w) d A}{m_{t}}
$$

where, $m_{b}$ and $m_{t}$ represent the reduction of mass flow rate caused by the blockage and the local total mass flow rate, respectively; $\bar{W}$ is the local mean axial velocity at the rotor outlet, and $w$ is the axial velocity in the blockage region; $A_{b}$ is the blockage region area. The blockage edge is recognized by a non-dimensional criterion

$$
\nabla_{w}=\left|\nabla_{r}\left[\frac{w}{\bar{w}}\right]\right|+\left|\nabla_{\theta}\left[\frac{w}{\bar{W}}\right]\right|
$$

which is defined by Zhang et al. [31]

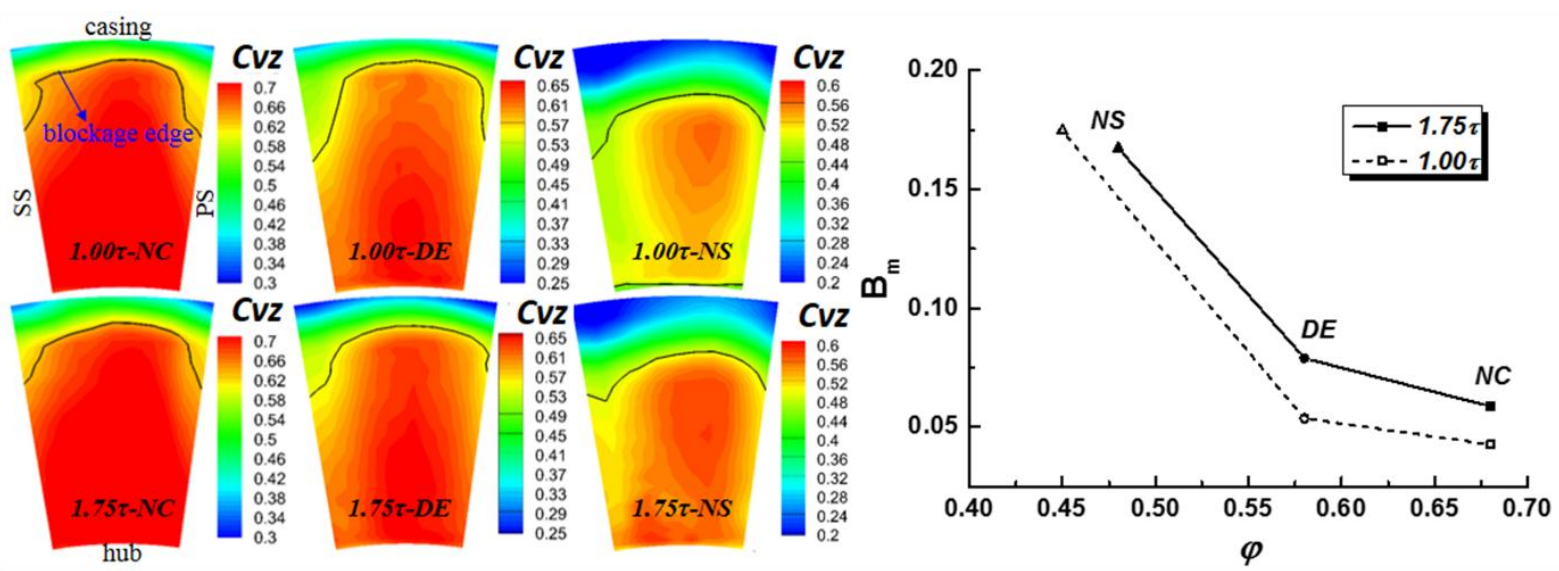

Fig. 17 Axial velocity fields at the rotor outlet and the corresponding rotor tip blockage coefficient.

The rotor tip blockage coefficient at the corresponding flow rate conditions is also shown in Fig. 17. The 
blockage of $1.75 \tau$ is significantly bigger than that of $1.00 \tau$ at the same flow rate condition. Blockage coefficient increases as the flow rate decreases. The increase is slow from NC to DE, but very fast from DE to NS. For $1.00 \tau$ and $1.75 \tau$, the variation of the blockage coefficient with the mass flow rate is fitted using a cubic polynomial function, as shown in Fig. 18. The variation for $1.50 \tau, 1.25 \tau, 0.75 \tau$, and 0.50 is obtained through linear interpolation, which is also shown in Fig. 18. The stable operating range of the compressor decreases with the increase of rotor tip gap size. Thus, the compressor surge line is plotted in this figure.

The unstable stage for $1.75 \tau$ on the compressor operating line happens around the NS condition $(\varphi=0.485)$. Thus, the critical blockage coefficient should be 0.153 . Critical blockage coefficient also can be obtained for other compressor configurations, as shown in Fig. 18. The variation of the critical blockage coefficient with the mass flow rate is almost linear. When a given point (mass flow rate coefficient as the abscissa, and rotor tip blockage coefficient as the ordinate) is located on the left of this line, the hub corner separation inside the stator passage will become an open type. When the given point is located on the right side of this line, the hub corner separation inside the stator passage will be a closed type. Due to the existence of the compressor surge line, the critical blockage coefficient line does not intersect with all rotor tip gap compressor configurations. For example, when the size of the rotor tip gap is bigger than $2.0 \%$ annulus height, open hub corner separation will not appear along the whole compressor operating line.

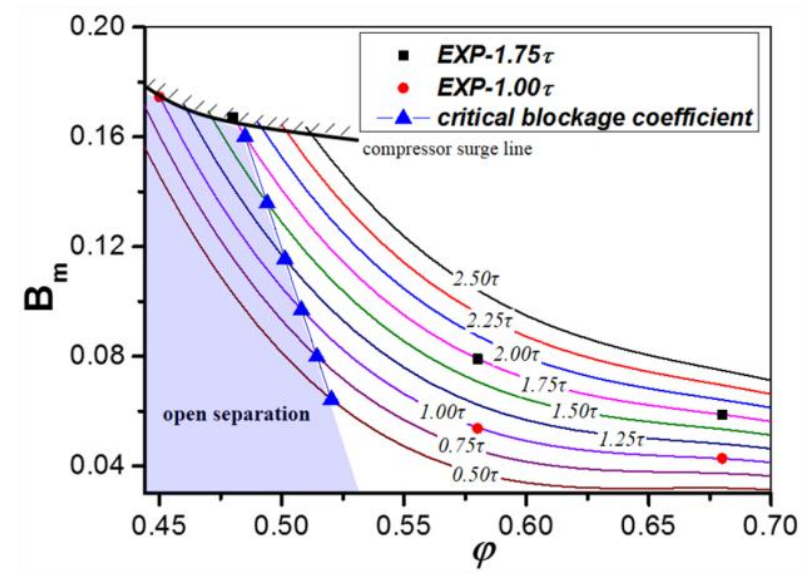

Fig. 18 Variation of the blockage coefficient with the mass flow rate.

\section{Conclusion}

This paper experimentally investigated a low-speed large-scale axial compressor with a highly loaded blade design. Six different rotor tip gaps were setup to investigate the effect of rotor tip gaps on the 3D separating 
flows inside the downstream stator. Measurements, including oil-flow visualization, five-hole probe, and SPIV, were conducted at several operating conditions. The conclusions drawn are:

(1) Four typical 3D flow structures exists inside the stator passage, namely, Type A, Type B, Type C, and Type D. Type A corresponds to small tip corner separation and small hub corner separation; Type B corresponds to big tip corner separation and small hub corner separation; Type C corresponds to small tip corner separation and big hub corner separation; Type $D$ corresponds to bigger hub corner separation without tip corner separation. These four flow structures will appear successively along the compressor characteristics as the flow rate decreases.

(2) Between Type $B$ and Type $C$, a short stage will occur in the compressor operating line, during which the flow field is very unstable. Before this stage, the 3D flow structure inside the stator passage is featured as Type B. After this stage, the 3D flow structure inside the stator passage is featured as Type $C$; the flow filed is very unstable during this stage and any small perturbation may prompt the transformation of the hub corner separation (from closed separation to open type).

(3) The effect of rotor tip gap on the flow in the following stator passage is mainly reflected in the redistribution of blade loading along the spanwise direction. Blade loading at the hub will increase with the decrease of rotor tip gap. Thus, hub corner separation increases and tip corner separation decreases. The variation of the corner separation scale is almost linear with the rotor tip gap size at small mass flow rate conditions.

(4) A critical rotor tip blockage usually happens during the transformation of corner separation types at a certain rotor tip gap configuration. The figure of the rotor tip blockage coefficient versus mass flow rate coefficient shows that the variation of the critical blockage coefficient with the mass flow rate is almost linear. When a given point (mass flow rate coefficient as the abscissa, and rotor tip blockage coefficient as the ordinate) is located on the left of this line, the hub corner separation inside the stator passage will be an open type. By contrast, when the given point is located on the right of this line, the hub corner separation inside the stator passage will be a closed type.

\section{Funding}

This work was supported by National Science Foundation of China, Grant Nos. 51376014, 51476004 and 51006007, and the Key Laboratory Fund No. 9140C410102140C41186. 


\section{References}

[1] C. Hah, J. Loellbach, Development of Hub Corner Stall and Its Influence on the Performance of Axial Compressor Blade Rows, Journal of Turbomachinery, 121 (1999) 67-77.

[2] Y. Dong, S.J. Gallimore, H.P. Hodson, Three-Dimensional Flows and Loss Reduction in Axial Compressors, Journal of Turbomachinery, 109 (1987) 354-361.

[3] V.-M. Lei, Z.S. Spakovszky, E.M. Greitzer, A Criterion for Axial Compressor Hub-Corner Stall, Journal of Turbomachinery, 130 (2008) 031006 (031010 pages).

[4] X.J. Yu, B.J. Liu, A Prediction Model for corner separation/stall in axial compressors, in: Proceedings of ASME Turbo Expo 2010: Power for Land, Sea and Air, Glasgow, UK, 2010.

[5] J.V. Taylor, R.J. Miller, Competing 3D Mechanisms in Compressor Flows, in: ASME Turbo Expo 2015: Turbine Technical Conference and Exposition, 2015.

[6] S.J. Gallimore, J.J. Bolger, N.A. Cumpsty, M.J. Taylor, P.I. Wright, J.M.M. Place, The use of sweep and dihedral in multistage axial flow compressor blading - Part II: Low and high-speed designs and test verification, Journal of Turbomachinery, 124 (2002) 533-542.

[7] D.C. Wisler, Loss Reduction in Axial-Flow Compressors Through Low-Speed Model Testing, ASME Journal of Engineering for Gas Turbines and Power, 107 (1985) 354-363.

[8] H.D. Schulz, H.E. Gallus, B. Lakshminarayana, Three-dimensional separated flow field in the endwall region of an annular compressor cascade in the presence of rotor-stator interaction. Part I. Quasi-steady flow field and comparison with steady-state data, Journal of Turbomachinery, 112 (1990) 669-678.

[9] H.D. Schulz, H.E. Gallus, B. Lakshminarayana, Three-dimensional separated flow field in the endwall region of an annular compressor cascade in the presence of rotor-stator interaction. Part II. Unsteady flow and pressure field, Journal of Turbomachinery, 112 (1990) 679-690.

[10] S.R. Wellborn, T.H. Okiishi, Effects of Shrouded Stator Cavity Flows on Multistage Axial Compressor Aerodynamic Performance, in: NASA-CR-198536, Iowa State University, 1996.

[11] N.R. Smith, N.L. Key, Vane Clocking Effects on Stator Loss for Different Compressor Loading Conditions, Journal of Propulsion \& Power, 31 (2014) 519-526.

[12] V. Gümmer, M. Goller, M. Swoboda, Numerical Investigation of End Wall Boundary Layer Removal on Highly Loaded Axial Compressor Blade Rows, Journal of Turbomachinery, 130 (2008) 011015-011015.

[13] N.M. McDougal, A Comparison Between the Design Point and Near-Stall Performance of an Axial Compressor, Journal of Turbomachinery, 112 (1990) 109-115.

[14] H.D. Schulz, H.E. Gallus, Experimental Investigations of the Three-Dimensional Flow in an Annular Compressor Cascade, Journal of Turbomachinery, 110 (1988) 467-478.

[15] X. Yu, Z. Zhang, B. Liu, The evolution of the flow topologies of 3D separations in the stator passage of an axial compressor stage, Experimental Thermal and Fluid Science, 44 (2013) 301-311.

[16] O. Domercq, J.F. Escuret, Tip clearance effect on high-pressure compressor stage matching, Proceedings of the Institution of Mechanical Engineers Part A Journal of Power \& Energy, 221 (2007) 759-767.

[17] M.A. Howard, P.C. Ivey, J.P. Barton, K.F. Young, Endwall Effects at Two Tip Clearances in a Multistage Axial Flow Compressor With Controlled Diffusion Blading, Journal of Turbomachinery, 116 (1994) 635-645.

[18] A.C. Foley, P.C. Ivey, Measurement of Tip-Clearance Flow in a Multistage, Axial Flow Compressor, Journal of Turbomachinery, 118 (1996) 211-217.

[19] N.R. Smith, N.L. Key, Flow visualization for investigating stator losses in a multistage axial compressor, Experiments in Fluids, 56 (2015) 1-17.

[20] B. Liu, S. Zhang, X. Yu, Experimental investigations on the effects of bowed stator in a highly loaded axial compressor stage, in: Fluid Machinery and Fluid Engineering, 2014 ISFMFE - 6th International Symposium on, 2014.

[21] H. Du, X.J. Yu, Z.B. Zhang, B.J. Liu, Relationship between the Flow Blockage of Tip Leakage Vortex and its Evolutionary Procedures inside the Rotor Passage of a Subsonic Axial Compressor, Journal of Thermal Science, 22 (2013) 522-531.

[22] B.J. Liu, G.F. An, X.J. Yu, Assessment of Curvature Correction and Reattachment Modification into the Shear Stress Transport Model within the Subsonic Axial Compressor Simulations, Proceedings of the Institution of Mechanical Engineers, Part A: Journal of Power and Energy, 229 (2015) 910-927.

[23] Z. Zhang, Investigations and Approximate Analyses for the Tip Leakage Flow in the Subsonic Axial Compressor Stage, in: PhD Thesis, School of Energy and Power Engineering, Beihang University, 2014.

[24] B.J. Liu, X.J. Yu, H.J. Yuan, H.X. Liu, H.K. Jiang, Y.T. Xu, Application of SPIV in Turbomachinery, Exp. Fluids, 40 
(2006) 621-642.

[25] L.H. Smith, The Effect of Tip Clearance on the Peak Pressure Rise of Axial-Flow Fans and Compressors, in: ASME Symposium on Stall, 1958.

[26] S. Baghdadi, Modeling Tip Clearance Effects in Multistage Axial Compressors, Journal of Turbomachinery, 118 (1996) 613-843.

[27] A.E. Perry, M.S. Chong, A Description of Eddying Motions and Flow Patterns Using Critical Point Concepts, Ann. Rev. Fluid Mech., 19 (1987) 125-155.

[28] A. Surana, O. Grunberg, G. Haller, Exact theory of three-dimensional flow separation. Part 1: Steady separation., Journal of Fluid Mechanics, 564 (2006) 57-106.

[29] A. Surana, G.B. Jacobs, G. Haller, Extraction of Separation and Attachment Surfaces from Three-Dimensional Steady Shear Flows, AIAA Journal, 45 (2007) 1290-1302.

[30] X.J. Yu, B.J. Liu, H.K. Jiang, Characteristics of the Tip Leakage Vortex in a Low-Speed Axial Compressor, AIAA Journal, 45 (2007) 870-878.

[31] Z. Zhang, X. Yu, B. Liu, Characteristics of the tip leakage vortex in a low-speed axial compressor with different rotor tip gaps, in: ASME Turbo Expo 2012: Turbine Technical Conference and Exposition, 2012, pp. 311-322. 